\title{
Visual analogue scale cannot measure postoperative pain in an objective fashion, on the contrary, pain vision can measure postoperative pain in an objective way.
}

\author{
MASASHI UCHIDA M. D. Anaesthesiology and Pain Clinic, Saitamaseikeikai Hospital, \\ Higashimatsuyama, Japan
}

Introduction: At present, the visual analogue scale (VAS) is the main method for objectively evaluating the degree of pain. This method is simple and it provides a standard for evaluating pain. However, the VAS consists of the patient' $s$ own subjective evaluations, so that there are large individual differences, and no quantitative analysis can be readily performed. In experimental trials, ketamine (ket) has been shown to reduce hyperalgesia, prevent opioid tolerance, and lower morphine consumption (1). However, in clinical trials in humans have shown mixed results in terms of effectiveness. To evaluate postoperative pain by using intravenous patient-controlled (IV-PCA) system with concomitant infusion of fentanyl (fen) and ket, we used VAS and a pain vision to evaluate postoperative pain.

Methods: Anesthesia was performed with AOPRfK (air-oxygen-propofol-remifentanil-low dose Ket: bolus infusion of $0.25 \mathrm{mg} / \mathrm{kg}$ followed by continuous infusion at the rate of $0.25 \mathrm{mg} / \mathrm{kg} / \mathrm{h}$ ) in orthopedic surgery. A PCA system was started after extubation. Patients were divided into three groups randomly and received different dosing regimens of co-administration of fen and ket by using PCA system. The infusion rate of fen was $0.5 \mu \mathrm{g} / \mathrm{kg} / \mathrm{h}$ in all groups. Group $\mathrm{KO}(\mathrm{n}=21)$ : patients were given only fen, Group K0.5(n=21): patients were given fen + ket $0.5 \mathrm{mg} / \mathrm{kg} /$ day, and Group K1. $0(n=21)$ : patients were given fen + ket $1.0 \mathrm{mg} / \mathrm{kg} /$ day. We evaluated patient's condition in the night on the day of surgery. Pain was measured by using VAS $(0-10,0$ : no pain, 10: worst possible unbearable pain) and degree of pain was measured by using PainVision ${ }^{\circledR}$ PS-2100 (OSACHI Co., Ltd., Nagano, Japan). Sedation Score was measured by using Richmond Agitation-Sedation Scale (RASS). Statistical analysis was performed by using Wilcoxon Singed-Rank Test in VASs, degrees of pain, and RASSs between GroupKO vs. GroupK0. 5, GroupK0 vs. GroupK1. 0, and Groupk0. 5 vs. Groupk1. 0. p<0. 05 was considered to be statistically significant. Because the study is not finished, the number of pairs of patients in each statistical analysis was not the same. We picked up patients in each analysis on a first-come-first served basis.

Pain Vision

(1) Current Perception Threshold

This mode evaluates the Minimum Perceived Current by gradually increasing the stimulating current. At the point the stimulating current is initially perceived, the hand switch button is pressed, ending the measurement and stimulating current generation. The point at which current is initially perceived represents the Minimum Perceived Current. (2) Pain Equivalent Current

After measurement of Current Perception Threshold, then use the same procedure to measure the level of current that produces a pain equivalent sensation. At the point the stimulating current is perceived as being equivalent in sensation to pain, the hand

switch button is pressed, ending measurement and stimulating current generation.

(3) The degree of pain $=100 \times$ ( pain equivalent current - current perception threshold) / current perception threshold
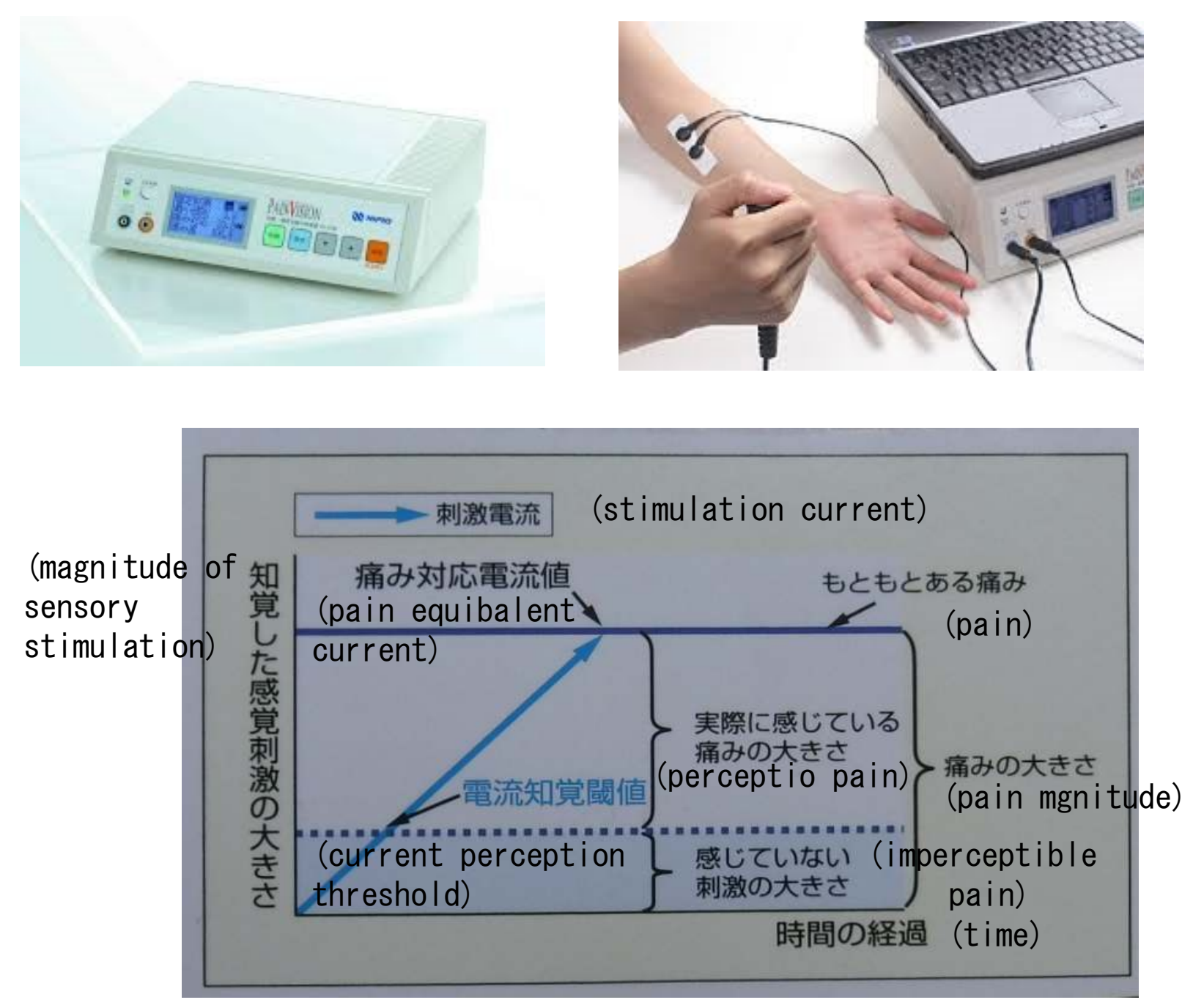

Results:

1) VAS: VASs were not significantly different among groups.

2) pain degree :

Group KO vs. GroupK0. 5 p $>0.05$,

Group K0 vs. GroupK1. 0 p $<0.01$,

Groupk0. 5 vs. Groupk1. $0 \mathrm{p}<0.01$.

3) RASS: RASSa were not significantly different among groups.

Discussion: Okuda $\mathrm{T}$ et al. (2) reported that VAS expresses factors include patient's psychological state and dissatisfactions at a given time, rather than simply evaluating actual pain in the treatment of acute phase of herpes zoster. Shikanai et al (1). used hot plate test to assess the analgesic effect of ketamine and/or morphine on thermal nociception. Pain vision is like hot plate test, because both are free from emotional interference. Most of works in clinical trials in humans used VAS for evaluating strength of pain. That seems to be the main reason for showing mixed results in terms of effectiveness.

Conclusions: VAS cannot measure postoperative pain in an objective fashion, on the contrary, pain vision can measure postoperative pain in an objective way.

References:

(1) H. Shikanai et al. Subanalgesic ketamine enhances morphine-induced antinociceptive activity without cortical dysfuction in rats. J. Anesth $2014 ; 28: 390-8$

(2) T. Okuda et al. A quantitative pain analysis for herpes zoster significance in comparison to the visual analogue scale (VAS)

(in Japanese) The Japanese Journal of Dermatology 2005;115: 2373-80 